\title{
Integrated Information System for Disaster Management: A Creative Case Study
}

\author{
Sabbir Ahmad 1 , Mohammad Ikhtear Rahman ${ }^{2}$, Avijit Mallik ${ }^{3,}$,, Arman Arefin $^{3}$ \\ ${ }^{1}$ Department of Computer Science \& Engineering, Rajshahi University of Engineering and Technology, Rajshahi, Bangladesh \\ ${ }^{2}$ Department of Economics, University of Dhaka, Dhaka, Bangladesh \\ ${ }^{3}$ Department of Mechanical Engineering, Rajshahi University of Engineering and Technology, Rajshahi, Bangladesh \\ Email address: \\ contact.jim13@gmail.com (A. Mallik) \\ ${ }^{*}$ Corresponding author
}

\section{To cite this article:}

Sabbir Ahmad, Mohammad Ikhtear Rahman, Avijit Mallik, Arman Arefin. Integrated Information System for Disaster Management: A Creative Case Study. International Journal of Sustainability Management and Information Technologies. Vol. 3, No. 5, 2017, pp. 46-52. doi: $10.11648 /$ j.ijsmit.20170305.11

Received: October 3, 2017; Accepted: October 24, 2017; Published: November 25, 2017

\begin{abstract}
Debacle reaction and recuperation endeavors require auspicious association and coordination of open crisis benefits so as to spare lives and property. Today, IT is utilized as a part of this field just to a restricted degree; however there is a huge potential for expanding productivity and viability in adapting to a calamity. In this paper we outline necessities and imaginative innovation for an incorporated fiasco administration correspondence and data framework, tending to specifically arrange, setup, planning and information administration issues amid the reaction and recuperation stages.
\end{abstract}

Keywords: Disaster Management, IT, Networking, Information System

\section{Introduction}

Normal and man-made debacles, for example, quakes, surges, plane accidents, elevated structure crumples, or major atomic office glitches, represent an ever-show test to open crisis administrations. So as to adapt to such fiascos in a quick and exceptionally planned way, the ideal arrangement of data concerning the circumstance is a fundamental pre-imperative. Police, fire divisions, general wellbeing, common resistance and different associations need to respond proficiently and separately, as well as in an organized way. This outcomes in the requirement for both intra and entomb association coordination at a few order levels [1]. Since coordination requires current data, and such data must be imparted upstream and downstream inside and between associations progressively, the need emerges for an incorporated correspondence and data framework for fiasco administration that gives proficient, solid and secure trade and handling of significant data. In this paper, reacting to a call of the German government, we recognize focal outline issues and building ideas for an incorporated catastrophe administration framework, giving a foundation that permits to even and vertical data spill out of the officer or fire fighter on the scene up to the focal operations staff by methods for a multi-level remote voice and information correspondence framework, and in addition coordinated applications that mirror the at present chose authoritative structure sufficient to the protect exertion. At all levels, we should accommodate recording and investigation of the present circumstance, self-loader information total and de-accumulation, asset planning, and access to administrations and databases. Systems incorporate earthbound trunked radio (as imagined for the European open wellbeing division) or satellite innovation for wide territory correspondence, remote LAN adhoc systems for catastrophe site problem areas, and individual or body range systems for cutting edge work force, enabling them to go about as information sources and sinks by methods for keen associated gadgets, e. g. powerful versatile terminals and sensors. Applications are to be planned around an adaptable data and work process idea in view of formats for quick adjustment to alterations of the hierarchical structure because of circumstance changes. Disseminated, excess, and versatility sufficient databases accommodate data get to even in questionable or low transmission capacity conditions through pre-getting and storing; benefit disclosure and auto- 
arrangement plans lessen the requirement for manual organization in furious circumstances. GIS and helpful order situations help operations staff in their offices or vehicles. We devise "data condition compactness" for included adaptability and versatile operations. While the concentrate of this paper is on calamity reaction (as characterized by FEMA, the US Federal Emergency Management Agency [5]) and recuperation, satisfactory readiness before a catastrophe strikes is indispensable. Chosen points to address in the pre-fiasco stage incorporate setting up recreations, (for example, clearings of structures) to be keep running with ebb and flow information should a debacle strike, and recognizing and connecting information assets for consistent access amid a crisis. In the most basic period of a catastrophe (i.e. in no time earlier and keeping in mind that it happens) activities vital for limiting harm or sparing lives ought to be performed, to the degree conceivable, naturally, e. g. shutting security valves, controlled shut down of electrical frameworks, programmed opening or shutting of crisis entryways and so on. Besides, an incorporated fiasco administration framework must have the capacity to give applicable information to a post-calamity lessons learned investigation and for preparing purposes.

The structure of this paper is as per the following: Following this presentation, we investigate client necessities and give, in segment 3, a design diagram. Area 4 tends to applications and data stream. Segment 5 portrays in our vision for wide, neighborhood, individual, and body range systems, and segment 6 talks about administration setup. Areas 7 and 8 address information administration and asset planning, separately. Segment 9 finishes up this paper.

\section{Client Requirements Analysis}

Fraunhofer Gesellschaft directed an investigation on catastrophe and crisis administration frameworks [11], asking specialists in the field what innovation they as of now utilized and what they missed. Aside from correspondence and data administration, the accompanying territories were tended to: advancement and recreation, choice help, perception, geographic data frameworks (GIS), and reenactment and preparing. One of the discoveries is that, in the surveyed specialists' assessment, keeping up correspondences is the "essential test" amid a debacle. Remarking on the best in class in correspondences and data administration, specialists expressed that the accompanying significant prerequisites were not yet met agreeably:

1. Integration and linkage of data

2. Availability of correspondence, repetition of connections

3. Fast information gets to

4. Timeliness and refreshing of data

5. Standardization of data

Broadening the extent of the examination from Germany to the United States, the effect of the 9/11 occasions on the needs in a fiasco administration was resolved. Prior to the psychological militant assaults, flooding, fire, tremor and risky material mishaps were considered to have "high need", with an innovative concentrate on nearby and versatile correspondence focuses, recreation and preparing, though after 9/11, against fear endeavors were doled out "most extreme need", including digital security, verification, picture handling, sensors, coordination, information administration, and preparing to the mechanical concentration territories [11]. Moreover, we surveyed fire offices for this outline consider. We allude to MESA [10] for necessities and exercises in the territory of portable correspondence institutionalization.

\section{Framework Architecture}

Figure 1 demonstrates an abnormal state perspective of the proposed correspondence engineering. Police, fire office and other administrations' headquarter (HQ) structures are associated with each other and with government specialists, e. g. the state senator, by earthbound and additionally satellite systems.

In like manner, when fiasco site summon posts are built up, they are associated by earthbound remote or satellite connections to the individual HQ. For "problem area" on location correspondences, a remote LAN (foundation, impromptu, or both) is set up. Firefighters and other staff may be furnished with individual or body zone systems, giving availability to sensors and terminal presentations. The approach taken in [9] is comparable, stressing quick "last mile" organization, lacking however the last "yard". The data stream of utilization can be both level, i.e. between peer substances, and vertical, i.e. along an association's chain of command and past; both push and force data proliferation are to be upheld.

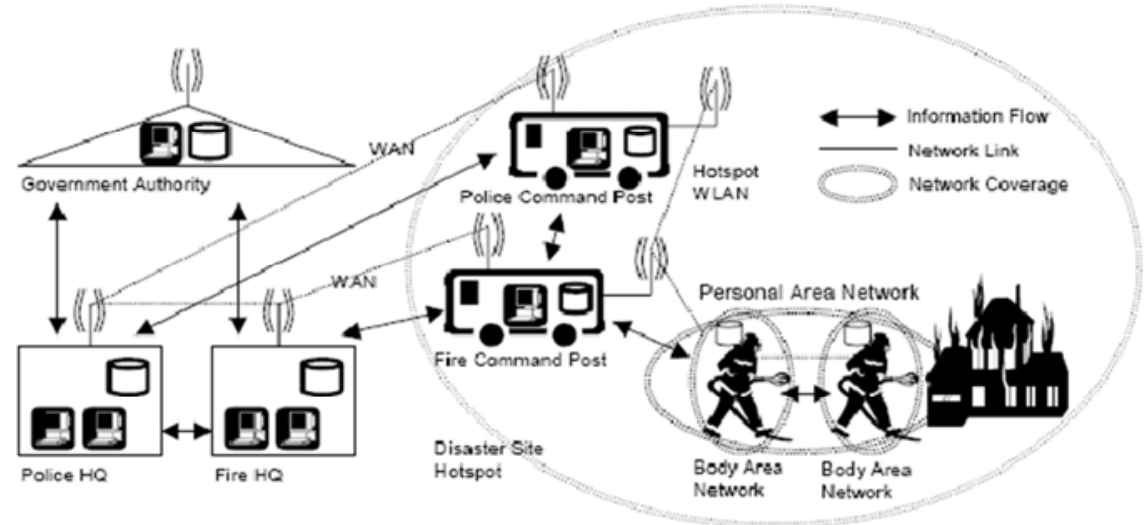

Figure 1. Communication architecture schematic. 


\section{Applications and Information}

In this segment, we adopt a base up strategy and first portray how cutting edge faculty like firefighters or other safeguard specialists working in troublesome territory may profit by the conceived framework. Firefighters' gear frequently incorporates sensors and finders, e. g. for radiation or dangerous gasses. The readings are generally transmitted by voice correspondences to squad pioneers. More prompt and solid information transmission can be refined utilizing shrewd sensors connected, by means of systems, to a PC in the squad pioneer's vehicle, where they are immediately examined and put in setting. Now and again, information might be coupled to the area of the estimation utilizing versatile GPS beneficiaries, so that, for instance, risk zones can be resolved all the more exactly. In addition, the crucial parameters of firefighters and the rest of the measure of pressurized air can be persistently transmitted for checking purposes [10]. In this way firefighters are information sources in our framework, yet they are likewise information sinks: Messages, perilous material notices, maps, and information on missing people might be transmitted to the strong cell phones they are furnished with. Obviously, disconnected operation ability is an unquestionable requirement in troublesome correspondence situations.

So as to adapt to a fiasco, all associations engaged with the safeguard exertion need to communicate nearly at different levels. These pecking order levels relate to accumulation levels at which assembled information is examined, placed in setting, and changed in reports (upstream) and directions (downstream). In the meantime, choices regularly must be taken in a specially appointed way, which requires proficient access to data supporting such choices, or, at times, an online "help work area". Consequently, from squad pioneers upward, our proposed applications enable gathered information to be connected with other data, to be totaled or de-collected, and to be traded with others as indicated by a data stream display considered proper for the circumstance. In like manner, a work process framework that, by utilizing formats and considering the included associations and the quantity of chain of importance levels, effortlessly adjusts to changing hierarchical structures encourages community oriented work inside and crosswise over administrations. Staff in central command frequently needs to play out a planning and coordination occupation, and they go about as an interface to different organizations and to the general population, so they are, because of their physical separation to the calamity site, especially reliant on cutting-edge data inflow. Then again, HQs for the most part have immense measures of put away information, e. g. on risky materials, which may should be gotten to by on location work force. This call for coordinated applications expanding on wide range information connects between the HQs and site charge posts. On the off chance that a fiasco spreads, even HQs may should be moved, or operations chiefs may choose to draw nearer to the scene, so it is imperative to give a "compact data condition" prepared for migration. This places extra prerequisites on databases and helpful conditions accommodated HQs.

\section{Communication Networks}

In this segment, we address wide territory interchanges and neighborhood correspondence inside a calamity site. On the off chance that there are extensive or various separate hazardous situations, the WAN likewise goes about as a spine connecting a few site problem areas.

\subsection{Wide Area Communications}

Indeed, even in some intensely industrialized nations, for example, Germany, general society security segment still uses simple voice just radio frameworks that give just a solitary communicate sort channel per association and area. Hence, radio information transmission is practically inconceivable today, and, because of the absence of prioritization or gathering call plans, over-burden and "mayhem noticeable all around" make correspondence troublesome and temperamental amid significant full-ready crisis circumstances.

The utilization of business remote phone systems has regularly been proposed, however is seen basic on the grounds that these systems are past the associations' control and tend to fall amid calamities because of overpowering interest from private clients, or because of framework harm.

Such systems are consequently satisfactory for unimportant applications or as reinforcement for committed open security frameworks.

European nations are currently in an official choice stage for the appropriation of earthbound trunked radio advanced voice and information systems following the TETRAPOL or ETSI TETRA 25 measures working at 380-400 MHz [12]. With the end goal of this paper, we don't have to recognize these contending frameworks, and we propose to utilize both of them as a WAN correspondence reason for our conceived catastrophe reaction framework. It will subsequently, for instance, profit by TETRA's gathering call, prioritization, and encryption capacities. Be that as it may, because of the constrained transfer speed $(<28.8 \mathrm{kbps})$, information administration must be precisely planned. Satellites are a strong option especially for high transfer speed applications, yet (at any rate for two-way interchanges) their huge expenses debilitate boundless utilize. Order post vehicles go about as doors between the WAN and problem area site systems.

\subsection{Hot Spot Communications}

Problem area correspondence in fiasco territories alludes in any event to two unique sorts of areas which can be ordered into basic and informative ranges as takes after:

Most basic zones: these are the focal spots of threat and the central focuses for halting or controlling significant parts 
of a debacle. Particularly in these most basic regions cutting edge faculty engaged with battling against the catastrophe need to focus however much as could be expected on the wellspring of the fiasco and are clearly in the most risky and basic circumstance. Consequently they should be educated quickly and immediately in the event that the circumstance raises and either ecological parameters approach basic limits or individual particular fundamental parameters wind up noticeably basic. Moreover they have to remain in contact with a boss group giving data accumulated from sources not straightforwardly accessible to the bleeding edge faculty.

Regardless all data must be given to these individuals without expecting them to physically associate with any sort of gadget. Data must be given naturally and mostly discourse controlled by means of reasonable show advances, amplifiers and different pointers.

\section{Service and Device Configuration}

We have so far contended that the proposed framework needs to have the capacity to oversee immense measures of information at all levels. Trading information continuously between the correct substances is a key test. The data stream must be controlled such that amid the fiasco the framework is hearty also, prepared to be broadened or rearranged effectively. This segment demonstrates that these prerequisites call for auto and self-arrangement of the gadgets and administrations in the manner.

\subsection{Motivation for Auto-Arrangement}

Without appropriate arrangement of hosts in systems, they are not ready to locate each other, or to speak with each other. Gadget design is along these lines of most extreme significance. This should be possible either statically or powerfully. Gadgets that are for all time associated with a controlled organized are typically allotted static system setup parameters by overseers [7]. Different gadgets that are joined to regulated systems can utilize dynamic system setup. The gadgets themselves must be arranged, i.e. all the vital parameters must be doled out to the host (gadget) by a system arrangement benefit. The system setup benefit thus likewise requires setup.

Be that as it may, in a correspondence and data framework gone for debacle recuperation, manual organization of organize has is illogical or incomprehensible. Consequently, programmed design of the hosts is attractive.

\subsection{Communication Spheres}

As beforehand proposed, there are three sorts of on-screen characters in the framework with respect to their level of portability:

1. Stationary performing artists: Police, fire, and so on. HQs, government experts, and even remote specialists or associations (if there should arise an occurrence of calamities influencing a few nations)

2. Semi-versatile on-screen characters: portable order posts.

3. Mobile on-screen characters: cutting edge faculty, e. g. firefighters.

The correspondence can be viewed as organized in a "organize in arrange" chain of importance too. The versatile on-screen characters may have a few sensors speaking with a handheld gadget. Generally, together the devices frame a little wearable system, or body territories organize. The correspondence to the closest portable performing artists can happen in an individual zone arrange.

As examined in segment 5, the catastrophe site is secured by a WLAN, worked by semi-portable performers. These, in turn, speak with stationary performers through a WAN.

\subsection{Configuration of Devices}

One part of the arrangement of cell phones is tending to parameters. Information sinks and information sources must know (i.e. be designed) with whom to convey. The gadget interface must be arranged with a exceptional address. Copy address assignments must be identified, and message impacts must be overseen. Ordinarily, a portable on-screen character will work (either physically or, then again naturally) sensor gadgets, which accumulate unique sorts of information. A few information, similar to the measure of hazardous gasses noticeable all around, is pertinent both for him by and by, as well with respect to the order post. Other information, for example, situating data, may not be critical for the portable on-screen character, yet rather for his bosses at the order post.

\subsection{Discovery of Services}

The disclosure of these administrations can be overseen in different ways. At the best progression layers (stationary and semi-versatile performing artists), the administration data administration can be directed utilizing a stationary administration broker.

Performers offering administrations can enroll them in the administration merchant, and in addition survey it for accessible administrations. For the versatile on-screen characters, a dynamic procedure for beginning what's more, working a (versatile) benefit merchant is an alternative. To adapt to the amazingly powerful circumstance, administrations recorded in this portable administration merchant have an essentially bring down enlistment lifetime, keeping in mind the end goal to accurately portray the system state. Then again, if the quantity of versatile performers at present in contact is underneath specific limit esteem, no administration merchant would be doled out by any stretch of the imagination. In this case, benefit data can be overseen and traded using multicast.

As sensor gadgets are turned on and off and unique performers come all through reach of each other, the rundown of accessible administrations for every individual on-screen character will most presumably continually be evolving. This reality, in conjunction with the measure of differing data administrations included, proposes a 
framework that progressively and consequently designs itself. I.e. the gadgets should on a solitary premise discover which administrations are accessible, which other performer to send its readings to, display and depict its own administrations to different gadgets in the region, etc. However much as could reasonably be expected of the design and overseeing of administrations must be robotized, with a specific end goal to encourage the assignments of the different on-screen characters. The objective is to continuously present the correct information to the correct on-screen character at the privilege time with as meager human intercession as would be prudent.

Also, an auto-setup empowered framework can likewise deal with the heap on the system, e. g. through coordinating movements to elective summons posts. In this way, correspondence "bottlenecks" can be dodged, which generally could demonstrate deadly if happening at the offbase time. We will return to this issue in segments 7 and 8 .

\section{Data Management}

As said in the past segments, conveyed applications for catastrophe administration need to manage temperamental correspondence conditions, low information transmission rates, and distinctive preparing and capacity abilities of the gadgets utilized. Consequently quality insurances can't be given for the correspondence. On the other hand, choices in the charge posts depend on data gotten from individuals working in the basic range. The other way around, individuals in such ranges follow up on directions given by the central command. For the two sides it is thus vital to get finish data, as inadequate data conveyance can bring about wrong choices or activities.

Besides, choices must be taken rapidly. This implies data and guidelines must be conveyed quickly. Therefore, as sketched out in the accompanying subsection, the primary difficulties for information administration in portable what's more, temperamental conditions, particularly in a fiasco circumstances, are dependability and execution.

\subsection{Challenges}

Dependability implies that the client dependably gets finish data of the most astounding conceivable portentousness. Fragmented data must be recognized and, if conceivable, must be asked for once more. Generally the application or client must be advised about the transmission disappointment. In less basic circumstances it may be conceivable to reuse data from past transmissions.

The second vital factor is the execution of the framework. Next to the application, the reaction time of the framework relies upon the data transfer capacity of the correspondence channel. Henceforth low transmission rates make it troublesome to convey e. g. complex maps inside a brief span. The information administration needs to ensure the proficient utilization of the correspondence channels. Besides, the reaction time of the framework ought to be generally free of the number of correspondence accomplices in the framework.

Moreover the information structures utilized for information trade must be adaptable in various ways. Sensors can give their information in restrictive arrangements. Consequently they must be changed sooner or later to the standard information structure.

This should be possible at the getting gadget or, if the gadget can't do this, the information must be exemplified into a standard message and changed at some other point.

Moreover, the similarity of various renditions of information constructions must be taken care of. Information patterns can change after some time if new forms of an application are grown, yet it is vital that gadgets with various application variants be as yet ready to convey.

\subsection{Suggested Approach}

To conquer the already recognized issue, XML [3] ought to be utilized as a standard information exchange design.

XML reports can contain all required data from basic messages to complex maps. Besides it is adaptable in the treatment of developing information structures. A hindrance of XML may be long label names and void area, expanding the report estimate. In any case, the astute choice of label names in the outline stage and pressure will lessen the archive estimate fundamentally.

The proficient use of correspondence channels depends on a constant and adjusted transmission of information to dodge correspondence tops. Subsequently shrewd reserving, pre-getting and determination of XML reports are the center innovation for the execution of versatile information administration. Storing takes into account powerful utilization of correspondence data transfer capacity by maintaining a strategic distance from re-transmission of generally static data. Savvy pre-getting and choice systems are utilized for convenient conveyance of complex area related data, e. g. maps of structures.

The dependability of the framework is expanded by repetitive capacity of XML reports on various gadgets (peers) [6]. Subsequently data must be reproduced in a shared way among close-by gadgets. This declines the likelihood of data misfortune on account of a correspondence disappointment in light of the fact that different gadgets can be utilized as a "data switch". The other way around, along these lines it is conceivable to keep away from the loss of critical sensor data.

For the proposed advances information uprightness and convenience of data are critical. A conveyed exchange administration, which is adjusted to the extraordinary needs in catastrophe administration, guarantees that directions or specialized portrayals are totally transmitted to the beneficiary. Notices of inadequate transmissions are important. The storing system needs to consider the opportuneness level of data, e. g. static maps must be refreshed less as often as possible than directions. 


\section{Scheduling Disaster Management Task Forces}

We now move the concentration from correspondences and information administration to asset booking and talk about how IT might help utilize assets proficiently to "take care of business."

\subsection{The Challenge}

While anticipation, readiness, discovery and appraisal of a calamity as a rule occur in stationary situations, response on a catastrophe requires to a high degree the coordination of versatile teams that are dispatched on area for fiasco administration.

An imperative test is the effective use of the conveyed (work force) assets as for the inquiry, where and when which asset is doled out to what errand. This is the issue of asset planning, the topic of asset portion regarding the present errands, their needs, and their shared conditions.

Here, it is likewise important to manage the teams' advance with their task (undertaking progress observing), keeping in mind the end goal to have the capacity to refresh the asset arranging (asset re-allotment) if essential. The difficulties are:

1. The current circumstance on area is seen straightforwardly just by the teams themselves.

2. Task powers must be permitted to adjust the needs of their appointed assignments to the present circumstance keeping in mind the end goal to take into consideration a quick response on capricious changes.

3. Several autonomous associations give teams, whose capacities to act adequately are commonly reliant, and whose accessibility is evolving.

Effective planning of debacle administration teams in view of auspicious on location information is vital for dodging the accompanying run of the mill issues:

1. Idling of assets in light of lacking assignments

2. Inadequate prioritization of a team's action as a result of lacking circumstance information at the team or at the coordination focus (because of missing nearby or worldwide information, separately)

3. Idling of assets due to an indulgent coordination process nearby (who will do what, when?)

4. Duplicate work (because of absence of coordination) The objective is to defeat these issues by supporting the asset administration and coordination forms with reasonable IT foundations. Such a framework must:

5. ensure a team part's capacity to act self-ruling by giving nearby booking ability

6. provide customized plans for team individuals that are adjusted to their individual circumstances, in light of the coordination focus' worldwide procedure

7. support checking and logging of the exercises of a team part, dynamic adjustment of his own timetable, and engendering to the operation community for joining into the worldwide technique
8. allow for observation and recording of circumstance realities and activity necessities by the on location teams and for spread to the operation focus

\subsection{Towards a Mobile Task Force Coordination Infrastructure}

While the idea of an IT-based coordination of fiasco administration teams itself isn't new, past methodologies, for example, [4] don't sufficiently address all or a portion of the difficulties talked about above. In Figure 2, we give a framework design, supporting both nearby and worldwide booking, and in addition neighborhood securing of circumstance actualities. On the structural level, a test is the circulated upkeep of the diverse information stores for actualities, errands, and calendars.

Other than a framework design, we require reasonably concrete but by and large material models for depicting catastrophe circumstances, assignments, and circumstance certainties. These models are an essential for empowering (self-loader) i.e., PC based, or PC helped) plan arranging.

At last, the test of the man-machine interface must be tended to - all things considered, the consideration of the team ought to be centered around the present alleviation jobs that needs to be done, not on the cooperation with the individual errand scheduler.

Our work on circumstance mindful individual versatile help and individual errand booking [2] [8] recommends that nearby planning methodologies can be made valuable for supporting the powerful activity of faculty in normal calamity administration circumstances. Nonetheless, a generous test for the future will be the improvement of models and designs that help a really circulated way to deal with team booking.

\section{Decentralized Architecture}

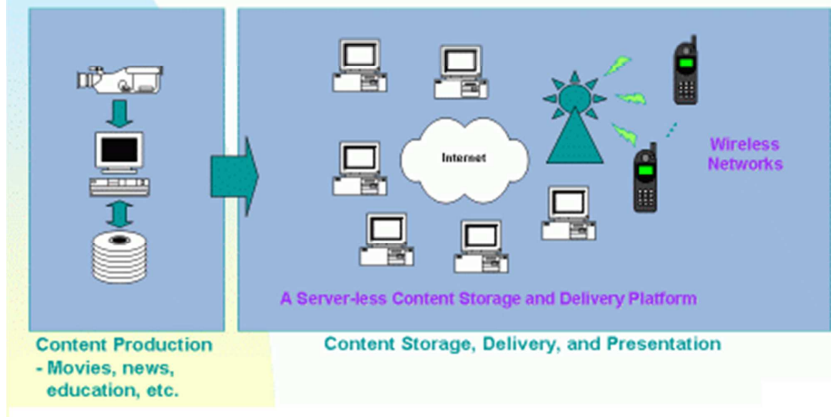

Figure 2. Decentralized architecture.

\section{Conclusion and Research Agenda}

In this paper, we distinguished difficulties and portrayed a coordinated correspondence and data framework for catastrophe reaction and recuperation, tending to specifically systems administration, administration and gadget arrangement, information administration, and asset booking.

So as to execute the depicted framework engineering, a 
few IT inquires about orders need to cooperate to give a powerful, yet simple to-utilize framework that helps crisis administrations adapt to catastrophes. To give some examples, organizing necessities to give vigorous correspondences at WAN, LAN, PAN, and BAN level, coordinating heterogeneous systems to enable the safeguard push to even in the most troublesome correspondence conditions.

Information administration must to give static and dynamic information where and when it is required. Security is a principal concern requiring answers for encryption, validation, information trustworthiness, and non-disavowal.

\section{Acknowledgements}

The idea and research was developed in the Research \& Development section of Appsplorer Technologies, Mirpur DOHS, Dhaka. All authors are truly indebted to Appsplorer and specially Md. Samdani Azad, Teaching Asst., Mahidol University, Thailand.

\section{References}

[1] Rodríguez-Espíndola, Oscar, Pavel Albores, and Christopher Brewster. "Disaster preparedness in humanitarian logistics: A collaborative approach for resource management in floods." European Journal of Operational Research (2017).

[2] Campigotto, Paolo, et al. "Personalized and situation-aware multimodal route recommendations: the FAVOUR algorithm." IEEE Transactions on Intelligent Transportation Systems 18.1 (2017): 92-102.
[3] Extensible Markup Language, https://www.w3.org/XML.

[4] Schöffel, Sebastian, and Johannes Schwank. "Analyzing Time-Dependent Infrastructure Optimization Based on Geographic Information System Technologies." International Conference on Applied Human Factors and Ergonomics. Springer, Cham, 2017.

[5] US Federal Emergency Management Agency, https://www.fema.gov.

[6] Bollen, Johan, et al. "An efficient system to fund science: from proposal review to peer-to-peer distributions." Scientometrics 110.1 (2017): 521-528.

[7] Moreno-Vozmediano, Rafael, et al. "Implementation and Provisioning of Federated Networks in Hybrid Clouds." Journal of Grid Computing (2017): 1-20.

[8] Wang, Chen, et al. "SEND: A Situation-Aware Emergency Navigation Algorithm with Sensor Networks." IEEE Transactions on Mobile Computing 16.4 (2017): 1149-1162.

[9] Erdelj, Milan, Michał Król, and Enrico Natalizio. "Wireless Sensor Networks and Multi-UAV Systems for Natural Disaster Management." Computer Networks (2017).

[10] Ehrig, Marcus, et al. "Reliable wireless communication and positioning enabling mobile control and safety applications in industrial environments." Industrial Technology (ICIT), 2017 IEEE International Conference on. IEEE, 2017.

[11] Weinke, Elisabeth, et al. "Land@ SlideHangrutschungsservice-Potenziale für einen CopernicusDownstream-Service."

[12] Bell, Michael GH. "Policy issues for the future intelligent road transport infrastructure." IEE Proceedings-Intelligent Transport Systems. Vol. 153. No. 2. IET Digital Library, 2006. 